\title{
Session 4: Inflammation and Related
}

\author{
Monday 7th November 2011. Moderator: James W. Larrick
}

\author{
[16.30-17.00] \\ 'New frontiers in inflammation therapeutics' \\ Alois B. Lang \\ GeNeuro, Geneva, Switzerland
}

Abstract not provided.

\section{[17.00-17.30]}

'CD44 and RHAMM (CD 168) non-identical twin players (and therapeutic targets) on the stage of inflammation therapy'

David Naor, Nathalie Assayag-Asherie, Shlomo Nedvetzki, Itmar Raz and Eva A. Turley

The Hebrew University, Jerusalem, Israel

CD44 and RHAMM are migration-supporting molecules in inflammatory and cancer cells. We have showed that targeting cell surface CD44 or RHAMM (receptor hyaluronic acid mediated motility or CD168) molecules, by anti-CD44 or anti-RHAMM monoclonal antibodies (mAbs) or by corresponding protein or gene vaccinations reduced or even eradicated the inflammatory activity in mouse models of rheumatoid arthritis (RA), multiple sclerosis and type 1 diabete (T1D) (Nedvetzki et al., PNAS, 101:18081-18086, 2004; Garin et al., J. Nerolog Sci 258:17-26,2007; Weiss et al., PNAS, 97: 285-290,2000). This approach is effective even when the targeting of the migration- supporting molecules was accomplished after the onset of disease (e.g., for T1D see Weiss et al., PNAS, 97:285-290, 2000). Using the collagen induced arthritis (CIA; the mouse analogue of RA) model, we have found that when CD44 is genetically deleted, RHAMM can compensate for CD44 in supporting in vitro cell migration and in vivo invasion of inflammatory cells into the inflamed joints (Nedvetzki et al., PNAS, 101:18081$18086,2004)$. In the NOD mouse model of T1D, unlike in CIA, CD44-null NOD mice show stronger resistance to the to the disease development than corre- sponding wild type mice, but the invasion of the inflammatory cells into the pancreatic islets was almost identical. These results suggest that RHAMM alone can support the invasion into the pancreatic islets (insulitis), but this event is essential, but not sufficient to induce T1D. We predicted that the generation of T1D is dependent on expression of CD44 on the insulin secreting cells, as cell surface CD44 can deliver, after activation, apoptotic signals, leading to program cell death. Indeed we have found in in vitro assay that glucose-stimulated pancreatic islet cells derived from wild type NOD mice showed reduced insulin secretion after incubation with a cocktail of pro-inflammatory cytokines. In contrast, the insulin secretion by corresponding cells from CD44-null mice is not influenced by the presence of pro-inflammatory cytokines, suggesting that CD44-null insulin secreting cells can resist the inflammatory attack by invading cells. In wild type NOD mice, unlike in CD44-null mice, RHAMM collaborates, or may even synergizes with CD44 to support invasion into the pancreatic islets. In summary, in NOD wild type mice RHAMM and CD44 cooprate in supporting inflammatory cell invasion into the pancreatic islets (but RHAMM can do the "job" alone if CD44 is missing). Yet, to generate T1D, the insulin secreting cells must express CD44 in order to be killed by the invading cells. In either case, RHAMM can be defined as a new therapeutic marker in T1D.

[17.30-17.50]

'A phage display approach for anti-inflammatory therapeutic antibody discovery'

Andrew E. Nixon

Dyax Corporation, Cambridge, MA, USA

Abstract not provided. 